\title{
Magnetization Measurements on Single Crystals of Superconducting $\mathrm{Ba}_{0.6} \mathrm{~K}_{0.4} \mathrm{BiO}_{3}$
}

\author{
Donavan Hall \\ National High Magnetic Field Laboratory \\ Florida State University \\ Tallahassee, FL 32306-4005 \\ R. G. Goodrich and C. G. Grenier \\ Department of Physics and Astronomy \\ Louisiana State University, Baton Rouge, LA 70803-4001 \\ Pradeep Kumar \\ Department of Physics \\ University of Florida, Gainesville, FL 32611-8440 \\ Murali Chaparala \\ Department of Physics \\ University of Virginia, Charlottesville, VA 22901 \\ M. L. Norton \\ Department of Chemistry \\ Marshall University, Huntington, WV 25755-2520
}

July 28, 2021

\begin{abstract}
Extensive measurements of the magnetization of superconducting single crystal samples of $\mathrm{Ba}_{0.6} \mathrm{~K}_{0.4} \mathrm{BiO}_{3}$ have been made using SQUID and cantilever force magnetometry at temperatures ranging between
\end{abstract}


1.3 and $350 \mathrm{~K}$ and in magnetic fields from near zero to $27 \mathrm{~T}$. Hysteresis curves of magnetization versus field allow a determination of the thermodynamic critical field, the reversibility field, and the upper critical field as a function of temperature. The lower critical field is measured seperately and the Ginzburg-Landau parameter is found to be temperature dependent. All critical fields have higher $\mathrm{T}=0$ limits than have been previously noted and none of the temperature dependence of the critical fields follow the expected power laws leading to possible alternate interpretation of the thermodynamic nature of the superconducting transition.

PACS numbers: 74.60.-w, 74.25.Ha, 74.25.Dw, 74.25.Bt

Accepted for publication in Philosophical Magazine B on 7 August 1999

\section{Introduction}

The discovery of high $\mathrm{T}_{c}$ superconductivity in $(\mathrm{La}, \mathrm{Ba})_{2} \mathrm{CuO}_{4}$ (Bednorz and Müller, 1986) was partly inspired by the bisthmuthate superconductor, $\mathrm{Ba}(\mathrm{Pb}$, Bi) $\mathrm{O}_{3}$. (Sleight, Gilson, and Bierstedt, 1975) Subsequent cuprate superconductors command the attention of a great many researchers because of their high $\mathrm{T}_{c} \mathrm{~s}$ (on the order of $100 \mathrm{~K}$ ). The discovery of high $\mathrm{T}_{c}$ superconductivity (HTSc) in the bismuthate system $\mathrm{Ba}_{1-x} \mathrm{~K}_{x} \mathrm{BiO}_{3}$ (BKBO) more than ten years ago(Cava, Batlogg, Krajewski, Farrow, Jr, White, Short, Peck, and Kometani, 1988; Mattheiss, Gyorgy, and Johnson Jr., 1988; Hinks, Dabrowski, Jorgensen, Mitchell, Richards, Pei, and Shi, 1988) with a $\mathrm{T}_{c}$ of $30 \mathrm{~K}$ began an equally significant, complementary course of research into the nature of HTSc for the following reasons: (1) it contains no copper, (2) it is an isotropic conductor, (3) it has no evident magnetic ordering off stoichiometry, (4) its superconductivity occurs at the boundary of a metal-insulator transition (a commonality with the cuprates) (Hinks, Dabrowski, Jorgensen, Mitchell, Richards, Pei, and Shi, 1988; Dabrowski, Hinks, Jorgensen, Kalia, Vashishta, Richards, a. Mark, and Mitchell, 1988; Sato, Tajima, Takagi. and Uchida, 1989), (5) normal state resistivity measurements evince other than pure metallic behavior (Dabrowski, Hinks, Jorgensen, Kalia, Vashishta, Richards, a. Mark, and Mitchell, 1988), (6) it has two related compounds: $\mathrm{Ba}_{1-x} \mathrm{Rb}_{x} \mathrm{BiO}_{3}$, that has a $\mathrm{T}_{c}$ of $\approx 29 \mathrm{~K}$ for $0.28 \leq \mathrm{x} \leq 0.44$ (Itti, Tomeno, 
Ikeda, Tai, Koshizuka, and Tanaka, 1991), and $\mathrm{Ba}(\mathrm{Pb}, \mathrm{Bi}) \mathrm{O}_{3}$, a more conventional superconductor with a $\mathrm{T}_{c}$ of $\approx 12 \mathrm{~K}$ (Batlogg, 1984), and (7) both $\mathrm{BKBO}$ and $\mathrm{Ba}(\mathrm{Pb}, \mathrm{Bi}) 0_{3}$ have a comparatively low density of states (low carrier densities), given their high values of $\mathrm{T}_{c}$.

There have been several previous measurements of the magnetic properties of superconducting $\mathrm{Ba}_{1-x} \mathrm{~K}_{x} \mathrm{BiO}_{3}$ reported in the literature (Batlogg, Cava, Rupp, Mujsce, Krajewski, Remika, Peck, a. Cooper, and Espinosa, 1988), (Welp, Kwok, Crabtree, Claus, Vandervoort, Dabrowski, Mitchell, Richards, Marx, and Hinks, 1988), (McHenry, Maley, Kwei, and Thompson, 1989), (Grader, Hebard, and Scheenmeyer, 1990), (Huang, Fang, Xue, Hor. Chu, Norton, and Tang, 1991). For $\mathrm{x}=0.4$, single crystal measurements of $\mathrm{H}_{c 1}$ were made by Grader, Hebard, and Scheenmeyer (1990) using an electrodynamic force balance technique on micron-sized single crystal samples. It was found that the lower critical field values measured on their near perfect single crystals were much higher $\left(250\right.$ gauss at $10 \mathrm{~K}$ ) than the $\mathrm{H}_{c 1}$ values reported by Batlogg et al. (1988) on powdered samples of similar composition (90 gauss at $10 \mathrm{~K}$ ). Further measurements of $\mathrm{H}_{c 1}$ on large single crystals have been done by Huang et al. (1991) who also reported low values of 95 gauss at $5 \mathrm{~K}$. All of these measurements were done on samples of imprecisely known demagnetization factors. For single crystals, in Grader et al. (1990), $\mathrm{H}_{c 1}$ is found to vary linearly with temperature over the range of temperatures measured $(7-22 \mathrm{~K})$ whereas in Huang et al. (1991) the $\mathrm{H}_{c 1}$ versus temperature curve shows a downward curvature as $\mathrm{T}=0$ is approached.

The upper critical field $\mathrm{H}_{c 2}$ also was reported in Batlogg et al. (1988), and extensive $\mathrm{H}_{c 2}$ studies of powdered samples of varying composition were done by Welp et al. (1988). In the constant-field, temperature-dependent magnetic measurements done by Batlogg et al. (1988) a linear negative slope of $\mathrm{dH}_{c 2} / \mathrm{dT}=-0.5 \mathrm{~T} / \mathrm{K}$ between 20 and $29 \mathrm{~K}$ was observed. Constantfield, temperature-dependent resistivity measurements of several potassium concentrations obtained by Welp et al. (1988), show that for $\mathrm{x}=0.4$, a slope of $-0.6 \mathrm{~T} / \mathrm{K}$ fit temperatures well below $\mathrm{T}_{c}$. A slight, positive curvature of $\mathrm{dH}_{c 2} / \mathrm{dT}$ was found near $\mathrm{T}_{c}$ in all of the samples measured in Welp et al. (1988). Recently, Samuely, Szabo, Jansen, Wyder, Marcus, Baril, and Escribe-Filippini (1996) have used tunneling measurements on low $\mathrm{T}_{c}$ samples $\left(\mathrm{T}_{c}=20 \mathrm{~K}\right)$ to determine $\mathrm{H}_{c 2}$ as a function of temperature; they find much lower values of $\mathrm{H}_{c 2}$ than in other measurements.

Several recent measurements of the magnetic properties of $\mathrm{Ba}_{1-x} \mathrm{~K}_{x} \mathrm{BiO}_{3}$ in Gatalskaya, Barilo, Shirayev, Szymezak, Szymezak, and Baran (1996) 
and Goll, Jansen, and Marcus (1996) and in an earlier paper by McHenry, Maley, Kwei, and Thompson (1989) have focused on the reversibility field as a function of temperature. In their study of flux creep in $\mathrm{Ba}_{0.6} \mathrm{~K}_{0.4} \mathrm{BiO}_{3}$, McHenry et al. (1989) found the reversibility field never to exceed $0.8 \mathrm{~T}$ down to $5 \mathrm{~K}$. In samples of nominal composition $(\mathrm{x}=0.34)$, Gatalskaya et al. (1996) find good agreement near $\mathrm{T}_{c}$ using a power law: $\mathrm{H}_{r} \approx\left(1-T / T_{c}\right)^{m}$ with $\mathrm{m}$ $=1.45$. For samples having a $\mathrm{T}_{c}$ of $20 \mathrm{~K}$, force magnetometer measurements over a temperature range of 0.4 to $20 \mathrm{~K}$ by Goll et al. (1996) show a continual upward curvature of $\mathrm{H}_{r}$ extending to $25 \mathrm{~T}$ at $0.4 \mathrm{~K}$. Thus different groups have measured widely different values for $\mathrm{H}_{r}$ on different samples.

We report extensive studies of the magnetization of single crystal samples of $\mathrm{Ba}_{0.6} \mathrm{~K}_{0.4} \mathrm{BiO}_{3}$. Magnetic field dependent hysteresis curves of the magnetization at constant temperature were recorded at temperatures ranging from 1.3 to $32 \mathrm{~K}$ in applied fields from zero to $27 \mathrm{~T}$, and the temperature dependence of the magnetization was measured from above room temperature down to below the superconducting transition temperature. From these measurements, we derive several quantities: (1) the complete temperature dependence of the lower critical field $\mathrm{H}_{c 1}$, (2) the upper critical field $\mathrm{H}_{c 2}$, (3) the reversibility field $\mathrm{H}_{r}$, and (4) the thermodynamic critical field $\mathrm{H}_{0}$. All of these critical fields are determined as a function of temperature. In addition, the temperature dependence of the normal state susceptibility is determined.

These results highlight the unique nature of the properties of $\mathrm{BKBO}$. While the qualitative features of the magnetic properties are quite reasonable, and look much like any other superconductor, in detail, BKBO has a remarkable number of anomalous properties. For example, in a conventional superconductor, both $\mathrm{H}_{c 1}$ and $\mathrm{H}_{c 2}$ have the same temperature dependence. Indeed the ratio, $H_{c 2} / H_{c 1}$, within logarithmic factors, is the square of the Ginzburg-Landau (GL) parameter, $\kappa$, and is temperature independent near $\mathrm{T}_{c}$. In $\mathrm{BKBO}, \mathrm{H}_{c 1}$ and $\mathrm{H}_{c 2}$ have different temperature dependences, leading to a temperature dependent (and divergent at $\mathrm{T}_{c}$ ) $\kappa$. The thermodynamic critical field $\mathrm{H}_{0} \propto\left(1-T / T_{c}\right)^{2}$ in contrast to a conventional superconductor where the exponent is one. The specific heat discontinuity at $\mathrm{T}_{c}$ appears to be zero. Likewise, we find the discontinuity in the magnetic susceptibility to be zero. When all of these results are taken together, it has recently been shown Kumar, Hall, and Goodrich (1999) that a new model for the thermodynamic nature of the phase transition from the normal to SC state gives good agreement with the results.

This paper is organized as follows: In section 2 we briefly describe the 
sample preparation technique following Norton and Tang Norton and Tang (1991). Section 3 contains a description of measurement techniques in detail. Section 4, divided into five subsections, goes through the results for $\mathrm{H}_{c 1}$, $\mathrm{H}_{0}, \mathrm{H}_{c 2}, \mathrm{H}_{r}$ and finally the normal state, temperature dependent magnetic susceptibility. Section 5 presents our current understanding of these results, relating them to earlier measurements. We also include a brief discussion of the properties of phase transitions of order $>2$, and how they relate to the results presented here. The paper ends with a summary of our conclusions in section 6 .

\section{Sample Preparation}

The crystals used in this study were grown by scaling up the electrosynthetic method described by Norton and Tang (1991). A typical procedure, briefly listing the essential modifications, is given here. An initial charge of 344 $\mathrm{g}$ of $\mathrm{KOH}$ (Baker, Reagent Grade) and $41 \mathrm{~g} \mathrm{Bi}{ }_{2} \mathrm{O}_{3}$ (Baker, Analyzed) was melted in a $300 \mathrm{ml}$ PFA transfer container (Berghof / America) using a temperature regulated (Omega) heating mantle (Glas-Col), with stirring, producing a solution saturated with bismuth ions. To this solution, $27 \mathrm{~g}$ of $\mathrm{Ba}(\mathrm{OH})_{2}$. $8 \mathrm{H}_{2} \mathrm{O}$ (Matheson, Reagent) was slowly added, with stirring, under an atmosphere of flowing water saturated nitrogen, an inert cover gas. After the solution clarified, and stabilized at $240{ }^{\circ} \mathrm{C}$, a $2 \mathrm{~mm}$ diameter (0.1mm wall thickness) platinum tubing cathode (Goodfellow, $99.95 \%$ ), a $3.2 \mathrm{~mm}$ diameter bismuth rod reference electrode (ESPI, 5N purity) and a $1 \mathrm{~mm}$ diameter silver wire anode (Aesar, $99.9 \%$ ) were inserted $1 \mathrm{~cm}$ into the solution. A potential of $0.69 \mathrm{~V}$ was applied utilizing a potentiostat (BAS model CV 27). Multiple crystals were harvested after 340 hours by extraction of the silver anode with the firmly attached crystals from the molten solution, followed by air cooling. Adhering solidified flux was removed by rinsing with distilled water. The samples were air dried, and the least flawed single crystals displaying cubic morphology were chosen for this study.

The high $\mathrm{T}_{c}(>30 \mathrm{~K})$ found for these crystals suggests that they were very close to the optimal superconducting stoichiometry. Subsequent $\mathrm{X}$ ray analysis showed the $1 \mathrm{~mm}^{3}$ facets used in these experiments were single perovskite crystals with a mosaic spread of less than $2^{\circ}$.

One of the problems in determining $\mathrm{H}_{c 1}$ has been the fact that the sample demagnetizing factor is of importance for this measurement. Without 
a controlled demagnetizing geometry, flux begins to penetrate the sample at different fields depending on position in the sample. For this reason we produced a single crystal sphere for the $\mathrm{H}_{c 1}$ measurements. Starting with a crystal of approximately $1.5 \times 1.5 \times 1.5 \mathrm{~mm}^{3}$ and a $\mathrm{T}_{c}$ of $30 \mathrm{~K}$ we ground it into a sphere in an air driven racetrack using a diamond sandpaper abrasive. The resulting sphere had a diameter of $1 \mathrm{~mm}$. After grinding the sphere, it was annealed in $\mathrm{O}_{2}$ for 24 hours at $400{ }^{\circ} \mathrm{C}$. Laue pictures showed no signs of strain.1 After this processing the sample used for the $\mathrm{H}_{c 1}$ measurements had a $\mathrm{T}_{c}$ of approximately $29 \mathrm{~K}$.

\section{Measurements}

The measurements near $\mathrm{T}_{c}$ reported here were made using a Quantum Design MPMS SQUID magnetometer $(\mathrm{B}<5.5 \mathrm{~T})$. Several precautions must be made in measurements on superconductors near $\mathrm{T}_{c}$ using a measurement system of this type. There are two basic problems. First, the magnetic moment of the sample holder can easily become larger than that of the sample near $\mathrm{T}_{c}$, and the maximum moment of the sample holder combined with the sample shifts along an axis parallel to the applied field. When this occurs, the output voltage from the SQUID pickup coils is not centered and spurious results in the calculation of the magnetic moment can occur. To overcome this problem, we held the samples in long quartz tubes having an inner diameter slightly smaller than the largest dimension of the sample to be measured. The empty quartz tube extends through all three pickup coils of the magnetometer during a complete vertical scan of the magnetometer. It was verified that the tubes produced a negligible signal over the scan length used. For most of the SQUID measurements, the samples were inserted into the tubes with the maximum dimension parallel to the axis of the tube, then turned slightly to hold them in place. Care was taken to center the sample in the pickup coils at each temperature because of the displacement due to thermal contraction of the quartz tube.

When the measurements on the spherical sample were done, the above procedure was not possible and it was secured to the inside of the quartz tube with a small piece of Kapton tape. This mounting limited the measurements to a minimum field of about $0.5 \mathrm{mT}$. The second problem with low field

\footnotetext{
${ }^{1}$ We are indebted to Larry Hults and J. L. Smith of Los Alamos National Lab for annealing and $\mathrm{x}$-raying this sample.
} 
SQUID magnetometry is that the superconducting magnet retains trapped flux at low fields. The trapped flux can be removed partially by cycling between positive and negative fields with decreasing final fields. This was done at each temperature at which a measurement for $\mathrm{H}_{c 1}$ was performed.

For temperatures between 2 and $32 \mathrm{~K}$ both constant temperature magnetic field hysteresis curves and constant field temperature hysteresis curves were recorded on two different crystals. At constant temperatures, measurements were taken as the field was raised from zero to a positive value greater than $\mathrm{H}_{c 2}$, then lowered through zero to a negative field value greater than $\mathrm{H}_{c 2}$. It was noted that any subsequent cycling of the field does not supply any additional information concerning the critical points. Hence, the symmetry of the complete hysteresis curves could be used to reduce the number of field points taken at a given temperature. An example of a complete constant temperature $(\mathrm{T}=22.5 \mathrm{~K}$ ) hysteresis curve is shown in Figure 1 with an expanded view showing $\mathrm{H}_{r}$ and $\mathrm{H}_{c 2}$ in the inset. The temperature of the sample was raised to $50 \mathrm{~K}\left(\mathrm{~T}>\mathrm{T}_{c}\right.$ at $\left.\mathrm{H}=0\right)$ before each constant temperature hysteresis measurement, then lowered to the measurement temperature in zero field. Thus, all constant temperature data reported are for zero field cooled samples. Samples not cooled in zero field would have a non-zero magnetization at zero field, but the magnetization curve for increasing field merges with the decreasing field portion at the same value of $\mathrm{H}_{r}$.

Further measurements of $\mathrm{H}_{c 2}$ and $\mathrm{H}_{r}$ were done using a cantilever force magnetometer at the National High Magnetic Field Laboratory (NHMFL) in a $27 \mathrm{~T}$ resistive magnet at temperatures from 1.4 to $19 \mathrm{~K}$ with the applied magnetic field being swept at a rate of $0.25 \mathrm{~T} /$ minute. The sample used for these measurements had a $\mathrm{T}_{c}$ of $30 \mathrm{~K}$. An example of the constant temperature magnetic hysteresis curve taken with the force magnetometer is provided in Figure 2 .

The constant field measurements were started at a temperature above $T_{c}$. The samples were cooled to $4.5 \mathrm{~K}$ in zero field, the field applied, the temperature slowly raised, point by point, to above $\mathrm{T}_{c}$ while recording magnetization, then again cooled to $4.5 \mathrm{~K}$ during the measurement in the applied field. An example of a complete cycle of this type of data is shown in Figure 3. Again, near $\mathrm{T}_{c}$ the magnetization is extremely small and care was taken to avoid the effects of the sample holder. Finally, we measured the magnetization at several temperatures at two constant fields in the normal state from 32 to $300 \mathrm{~K}$ to determine the normal state susceptibility. 


\section{Results}

In addition to the separate $\mathrm{H}_{c 1}$ measurements, there are several critical fields to be obtained from the hysteresis data: $\mathrm{H}_{0}, \mathrm{H}_{c 2}$, and $\mathrm{H}_{r}$. The thermodynamic critical field $\mathrm{H}_{0}$ is obtained only from the constant temperature data while $\mathrm{H}_{c 2}$ and $\mathrm{H}_{r}$ were measured both at constant temperature and constant field. None of the critical fields can be determined in a straightforward manner and we state in detail how we have extracted them from the data. Between approximately 18 and $32 \mathrm{~K}$, the two highest critical fields were determined using the SQUID magnetometer. Below $18 \mathrm{~K}, \mathrm{H}_{c 2}$ was in excess of the maximum field of the SQUID magnetometer $(5.5 \mathrm{~T})$. Above $22 \mathrm{~K}, \mathrm{H}_{c 1}$ was sufficiently low that no reliable data could be obtained.

\section{$4.1 \quad \mathbf{H}_{c 1}$}

Values of $\mathrm{H}_{c 1}$ reported here were obtained from the spherical sample from 4.2 to approximately $22 \mathrm{~K}$. Typical low temperature, low field magnetization curves for several temperatures are shown in Figure 4 . The initial slope of the curve is given by $\mathrm{M} / \mathrm{H}=\mathrm{V} / 4 \pi(1-\mathrm{D})$, where $\mathrm{V}$ is the volume of the sample and $\mathrm{D}$ is the demagnetization factor. Only when the sample geometry is such that $\mathrm{D}=0$ will this constant slope extend to $\mathrm{H}_{c 1}$, at which point the magnetization would abruptly decrease. When $\mathrm{D} \neq 0$, the field begins to penetrate the sample at different points gradually until the entire sample is in the mixed state. We shaped a single crystal sample into a sphere, (D $=1 / 3$ ), hence the initial slope is much larger and the transition from the Meissner to the mixed state much sharper than for the crystals that have non-symmetric shapes.

To determine the value of $\mathrm{H}_{c 1}$, the following procedure was used. The low field, linear portion of the curve was fit with a straight line, and the field value at which the measured magnetization deviated from the straight line was taken to be $\frac{2}{3} \mathrm{H}_{c 1}$. Values obtained from this fit for $\mathrm{H}_{c 1}$ are plotted as a function of temperature in Figure 5. Note that below $15 \mathrm{~K}$ these results give the same values as those reported in Grader et al. (1990).

\section{$4.2 \quad \mathrm{H}_{0}$}

The thermodynamic critical field is obtained by integrating over half the cycle, $\mathrm{M}_{R}(\mathrm{H})=\left[\mathrm{M}\left(\mathrm{H}_{\text {incr }}\right)+\mathrm{M}\left(\mathrm{H}_{\text {decr }}\right)\right] / 2$, from zero to $>\mathrm{H}_{c 2}$. The value of 
$\mathrm{H}_{0}$ is obtained from:

$$
\int M \cdot d H=\frac{H_{0}^{2}}{8 \pi}
$$

Since SQUID magnetometry measurements require a constant applied field during the measurement, and the data were accumulated point by point we have numerically integrated the hysteresis curves to obtain $\mathrm{H}_{0}$ at each temperature. The temperature dependence of $\mathrm{H}_{0}$ is shown in Figure 6 for the sample used in the SQUID measurements. We have elected not to calculate values for $\mathrm{H}_{0}$ from the high field-low temperature data until the anomalous "fishtail" structure (see below), also noticed by Gatalskaya et al. (1996), is fully understood. The fishtail adds area below the magnetization curve; thus, thwarting any attempt of straightforward analysis.

\section{$4.3 \quad \mathbf{H}_{c 2}$}

A typical plot of the magnetization for increasing field from zero tesla to above $\mathrm{H}_{c 2}$ is shown in Figure 1. There are several points about this curve that make determination of $\mathrm{H}_{c 2}$ complicated. Between $\mathrm{H}_{c 1}$ and $\mathrm{H}_{c 2}$ the curve is never linear in applied field, and an extrapolation to $\mathrm{H}_{c 2}$ from a linear portion of the curve in the superconducting state near $\mathrm{H}_{c 2}$ is not possible (Hao, Clem, McElfresh, Civale, Malozemoff, and Holtzberg, 1991). Above $\mathrm{H}_{c 2}$ BKBO is diamagnetic (mainly due to the atomic core contribution to the magnetization) and the total magnetization never becomes positive upon passing from the superconducting to the normal state. To obtain a consistent value for $\mathrm{H}_{c 2}$, we have fit the linear diamagnetism data in the normal state above $\mathrm{H}_{c 2}$ to a straight line and taken the field at which the magnetization deviates from the extrapolation of this line to zero field to be $\mathrm{H}_{c 2}$. From values of $\mathrm{H}_{c 2}$ obtained in this manner, we show the temperature dependence of $\mathrm{H}_{c 2}$ in Figure 7 along with the determination of $\mathrm{H}_{c 2}$ from the constant field data to be discussed below. The minimum in the magnetization curve in this field region is fairly broad for all of the samples, but this technique gives values of $\mathrm{H}_{c 2}$ consistent with the ones that are obtained from temperature dependent data at constant field (Batlogg et al., 1988; Huang et al., 1991).

We observe the anomalous "fishtail" structure in our force magnetometer measurements also reported by Gatalskaya et al. (1996). These structures are not observed in the magnetization data taken with the SQUID magnetometer. This leads us to question the origin of these fishtails, specifically, whether they might be due to the measurement technique. The fishtails seen 
in our force magnetometer measurements might be due to a magnetic field sweep rate that exceeds the flux lattice relaxation time, or may be associated with uncompensated torque contributions from the sample. It should be noted that these fishtail structures do not affect our ability to determine $\mathrm{H}_{r}$ and $\mathrm{H}_{c 2}$ from these data, and these two critical fields are the only information we have extracted from the force measurements.

Using the $21 \mathrm{~K}$ values of $\mathrm{H}_{c 1}$ and $\mathrm{H}_{c 2}$, the GL parameter, $\kappa$, was calculated from the relation

$$
\frac{\ln \kappa}{2 \kappa^{2}}=\frac{H_{c 1}}{H_{c 2}} .
$$

We find that the value of $\kappa$ at $21 \mathrm{~K}$ is approximately 64 . This compares favorably with the value reported by Kwok, Welp, Crabtree, Vandervoort, Hulscher, Zheng, Dabrowski, and Hinks (1989) of $\kappa=59$.

\section{$4.4 \mathbf{H}_{r}$}

At temperatures above $15 \mathrm{~K}$, we are able to determine the value of applied field at which the hysteretic behavior becomes reversible, $\mathrm{H}_{r}$. We have taken this field to be the lowest value of $\mathrm{H}$ at which the increasing and decreasing applied field have the same measured values of $\mathrm{M}$. The temperature dependence of $\mathrm{H}_{r}$ is shown in Figure 7 .

\subsection{Temperature Dependent Constant Field Data}

Measurements of the temperature dependence of the magnetization at constant field give the same information in the $\mathrm{T}-\mathrm{H}$ plane through measurements of critical temperatures, $\mathrm{T}_{c 2}$ and $\mathrm{T}_{r}$, as do the constant temperature field dependent measurements. A view of one set of data taken at constant field is shown in Figure 3. The temperature at which the magnetization deviates from a constant on the high temperature side is taken to be $\mathrm{T}_{c 2}$. The temperature at which the increasing and decreasing measurements deviate is $\mathrm{T}_{r}$. One can see from the data presented in the inset of Figure 7 that the two different measurements give the same result. This agreement shows that the phase boundaries between the normal and superconducting states and between the reversible and irreversible flux regions within the superconducting states are equilibrium thermodynamic boundaries and are not controlled by non-equilibrium phenomenon. 


\subsection{Normal State Susceptibility}

The normal state susceptibility, $\chi$, was calculated from the difference of two magnetization curves taken at $0.3 \mathrm{~T}$ and $5.0 \mathrm{~T}$ at temperatures from $31 \mathrm{~K}$ to $350 \mathrm{~K}$ and is shown in Figure 8 with $\chi$ as a function of $1 / \mathrm{T}$ in the inset. The difference between the magnetization measurements, $\Delta \mathrm{M}$, divided by the field difference, $\Delta \mathrm{H}$, was taken to be the susceptibility, $\chi=\Delta M / \Delta H$. The reason for the large field difference for the two magnetization measurements is that $\mathrm{M}$ is small in each case, and the large $\Delta \mathrm{H}$ reduces errors in $\Delta \mathrm{M}$. As long as the magnetization remains in the linear response regime, the relatively large size of $\Delta \mathrm{H}$ is of no concern. The quartz tube sample holder contributes a small signal to the magnetization; this has been measured and subtracted from the difference magnetization.

\section{Discussion}

The temperature dependence and the magnitudes of the critical fields measured here are different from that expected from an ordinary type-II superconductor. In general, the critical fields have different temperature dependences from what is expected and some new phenomena are observed. We begin with a discussion of the normal state susceptibility.

In their initial measurements of the normal state susceptibility, Cava et al. (1988) found large extraneous paramagnetic contributions presumedly due to the presence of unreacted $\mathrm{KO}_{2}$ in their samples. In a following paper (Batlogg et al., 1988), the same authors corrected their data with an estimate of the core diamagnetism $\left(\chi_{c} \approx-7.5 \times 10^{-5} \mathrm{emu} / \mathrm{mol}\right)$ which is the same value used here. From their energy band calculations Mattheiss and Hamann (1988) determined the density of states at $\mathrm{E}_{F}$ in $\mathrm{Ba}_{0.6} \mathrm{~K}_{0.4} \mathrm{BiO}_{3}$ to be 0.46 states $/ \mathrm{eV}$. Using this value, we calculate the theoretical contribution to the susceptibility due to Pauli paramagnetism to be $2.7 \times 10^{-5} \mathrm{emu} / \mathrm{mol}$. Our measurements show that the normal state susceptibility at $\mathrm{T}=300 \mathrm{~K}$ is $-5.3 \times 10^{-5} \mathrm{emu} / \mathrm{mol}$ (see Figure 8), and this value agrees with that reported by Hundley et al. (1989) and Uwe et al. (1996) for a potassium concentration of $\mathrm{x}=0.40$. Our measurements show an increase in the normal state paramagnetism below $150 \mathrm{~K}$. We have fit the data below $150 \mathrm{~K}$ to a Curie-Weiss function plus a temperature independent Pauli term,

$$
\left(\chi-\chi_{\text {core }}\right)=\chi_{P}+\frac{C}{T-\Theta},
$$


where $\chi_{\text {core }}$ is the core diamagnetism, $\chi_{P}$ is the Pauli term, $C$ is the Curie constant, and $\Theta$ is the ordering temperature. The values obtained from the fits are $\chi_{P}=1.81 \times 10^{-5} \mathrm{emu} / \mathrm{mol}, C=8.87 \times 10^{-4} \mathrm{erg} \mathrm{K} / \mathrm{G}$, and $\Theta=2.48$ $\mathrm{K}$ for one sample and $\chi_{P}=1.81 \times 10^{-5} \mathrm{emu} / \mathrm{mol}, C=8.28 \times 10^{-4} \mathrm{erg} \mathrm{K} / \mathrm{G}$, and $\Theta=2.21 \mathrm{~K}$ for a second sample. Using the values of $C$ and assuming the impurities have a spin quantum number of $\frac{1}{2}$, the magnetic impurity concentration is estimated to be $0.66 \%$ and $0.71 \%$ for the two samples. If the impurities have a larger spin quantum number, then the magnetic impurity concentrations would be lower. The Pauli term for both samples is the same and is approximately $2 / 3$ of that obtained from band theory (Mattheiss and Hamann, 1988). We point out that we have interpreted the linear $1 / \chi$ vs. T behavior at low temperatures as being due to localized spin impurities. However, this behavior might also arise from other mechanisms intrinsic to the normal state.

If an overall linear fit to the $\mathrm{H}_{c 1}$ data is done, a slope of $\mathrm{dH}_{c 1} / \mathrm{dT}=-17.2$ $\pm 0.66 \mathrm{G} / \mathrm{K}$ is obtained as compared to $-11 \mathrm{G} / \mathrm{K}$ from Grader et al. (1990). These values are much larger than the value of Batlogg et al. (1988) of $-4.5 \pm$ $0.5 \mathrm{G} / \mathrm{K}$; also, the values of $\mathrm{H}_{c 1}$ derived from single crystals are larger than the powder samples used by Batlogg et al. From this linear fit to the $\mathrm{H}_{c 1}$ data, we find that $\mathrm{H}_{c 1}(\mathrm{~T}=0)$ is $393 \pm 6 \mathrm{G}$. However, it is clear that a linear function does not represent best the $\mathrm{H}_{c 1}$ dependence on temperature. Between 5 and $15 \mathrm{~K}$ the data appears to be linear. Around $15 \mathrm{~K}, \mathrm{H}_{c 1}(\mathrm{~T})$ experiences an inflection point and has a different curvature at higher temperatures. Attempts were made to measure $\mathrm{H}_{c 1}$ at fields approaching $\mathrm{T}_{c}$, but above $22 \mathrm{~K}$ the magnetization signal was too small to resolve with the SQUID measurement system near zero applied field.

We find that a good fit to the $\mathrm{H}_{c 1}$ data above $12 \mathrm{~K}$ is obtained from $\mathrm{H}_{c 1}(T) \propto\left(1-T / T_{c}\right)^{3}$ with the curve passing through the $\mathrm{H}=0$ value of $\mathrm{T}_{c}$ $=29 \mathrm{~K}$. The very low value of $\mathrm{H}_{c 1}$ is the reason the very broad temperature dependent magnetic transitions are observed in BKBO, even though the zero field resistive transitions are sharp. All of the magnetic measurements are made in small magnetic fields and continuous changes in $\mathrm{M}(\mathrm{T})$ are observed until the temperature is reached where $\mathrm{H}_{c 1}$ is greater than the measurement field. We note that $\mathrm{H}_{c 1}$ is the only measured thermodynamic critical field that does not have upward curvature as the temperature goes to zero.

As a result of our upper critical field measurements, we found that a power law fit where $\mathrm{H}_{c 2}$ and $\mathrm{H}_{r} \sim\left(1-\mathrm{T} / \mathrm{T}_{c}\right)^{m}$ yielded values of $\mathrm{m}=1.58$ and 1.97 for $\mathrm{H}_{c 2}$ and $\mathrm{H}_{r}$ respectively (as shown in Figure 9). Gatalskaya 
et al. (1996) found for $\mathrm{H}_{r}$ that $\mathrm{m}=1.45$ for a sample with a potassium concentration of 0.34. Also, Goll et al. (1996) report a value of $\mathrm{m}=1.5$ for $\mathrm{H}_{r}$ in their below optimum potassium concentration sample. Our samples had a nominal potassium concentration of 0.40 .

Welp et al. (1988) report upper critical field slopes for several concentrations of $\mathrm{K}$, but no positive curvature of the $\mathrm{H}_{c 2}$ versus temperature was reported. Like Affronte et al. (1994) and Gantmakher et al. (1996), we observe a positive curvature in the $\mathrm{H}_{c 2}$ versus temperature curve; however, we observe an enhanced curvature as compared to the results of Gantmakher et al. (1996). This curvature deviates significantly from the universal behavior predicted by Werthamer, Helfand, and Hohenberg (WHH) (Werthamer. Helfand, and Hohenberg, 1966) for superconductors with weak electronphonon coupling. Gantmakher et al. (1996) find empirically that their $\mathrm{H}_{c 2}$ data fits the function

$$
H_{c 2}(T)=32.2-1.8 T+0.025 T^{2} .
$$

A plot of this equation is shown in Figure [7. Our $\mathrm{H}_{c 2}$ data show a greater curvature than the fit of Gantmakher et al. (1996) and it appears a second order fit greatly underestimates the probable value of $\mathrm{H}_{c 2}(\mathrm{~T}=0)$, and their value of $32.2 \mathrm{~T}$ might be too low. Gantmakher et al. (1996) comment that the positive curvature is enhanced by disorder in the sample; therefore, the enhancement of the curvature and correspondingly in $\mathrm{H}_{c 2}(\mathrm{~T}=0)$ may be due to an intrinsic disorder in BKBO.

From our determination of $\mathrm{H}_{r}$ we observe that the irreversibility line also deviates from WHH theory and displays a positive curvature. Similarly, Gol] et al. (1996) observe a positive curvature in $\mathrm{H}_{r}$ versus temperature, and again our values of $\mathrm{H}_{r}$ are enhanced when compared with the values of Goll et al. (1996). Similarly, the thermodynamic critical field, $\mathrm{H}_{0}$, has a positive curvature as is shown in Figure 6 .

Samuely et al. (1996) have determined the upper critical field $\mathrm{H}_{c 2}$ by Andreev reflection in point-contact junctions, a measurement of the superconducting density of states. They find no positive curvature of the $\mathrm{H}_{c 2}$ versus temperature curve and claim that their measurements confirm an adherence of BKBO to WHH theory. This contradicts all other magneto-transport measurements (Affronte, Marcus, Escribe-Filippini, Sulpice, Rakoto, Broto, Ousset, Askenazy, and Jansen, 1994) that uniformly show $\mathrm{H}_{c 2}$ and $\mathrm{H}_{r}$ as a function of temperature in BKBO deviate from WHH theory. The only remarkable difference between the samples used by Samuely et al. (1996) and 
other groups is that their samples had a $\mathrm{T}_{c}$ of $\approx 23 \mathrm{~K}$. Studies, including this one, that use samples with $\mathrm{T}_{c} \mathrm{~s}$ of 30 to $32 \mathrm{~K}$ generally show a deviation from the WHH theory.

Measurement of magnetization across a phase transition may contain fluctuation effects. Following early works of Aslamazov and Larkin (1968), Ullah and Dorsey (1991) have proposed a scaling equation for temperature and field dependence of magnetization. The scaling variables used are

$$
\begin{array}{ll}
{\left[T-T_{c}(H)\right] /[H T]^{2 / 3}} & D=3 \\
{\left[T-T_{c}(H)\right] /[H T]^{1 / 2}} & D=2
\end{array}
$$

where the dimensionality of the electron system is given by D. For either $\mathrm{D}=3$ or $\mathrm{D}=2$ the magnetization is expected to follow a universal curve. The essential feature is that a smooth cross over takes place from a normal paramagnetic (or diamagnetic in the case of $\mathrm{BKBO}$ ) state to the mixed state of a superconductor. Thermodynamic quantities such as the magnetization and specific heat for YBCO have been shown to fit a 3D universal curve by Welp et al. (1991) The results of our $\mathrm{H}_{c 2}$ data being analyzed in this manner are shown in Figure 10. One can see that the data for magnetization collapse onto a universal curve. However, given that BKBO is a cubic isotropic system, it is surprising that our results are just as well described by $\mathrm{D}=2$ or $\mathrm{D}=3$. It appears that fluctuation theory cannot distinguish between 2 and 3 dimensional behavior in this case. Therefore, the broadening of the transition may be due to effects other than fluctuations.

Several studies of the specific heat of BKBO have been done (Hundley, Thompson, and Kwei, 1989; Graebner, Scheenmeyer, and Thomas, 1989; Stupp, Reeves, Ginsberg, Hinks, Dabrowski, and Vandervoort, 1989; Alba, 1992). All but one study (Graebner et al., 1989) ) conclude that $\Delta C_{p}$ is zero across the transition. Because of the large phonon contribution to the specific heat and the low density of electronic states, it is difficult to extract the comparatively small value of the electronic specific heat change across the transition. A calculation by Kwok et al. (1989) shows the BCS discontinuity in BKBO to be $\Delta \mathrm{C}_{p} / \mathrm{T}_{c} \approx 3.75 \mathrm{~mJ} / \mathrm{mole} \mathrm{K}^{2}$ or $\Delta \mathrm{C}_{p}=120 \mathrm{~mJ} / \mathrm{mole} \mathrm{K}$ if $\mathrm{T}_{c}$ $=32 \mathrm{~K}$. This value is calculated from the relation

$$
\Delta C_{p} / T_{c}=\left(\frac{1}{8 \pi \kappa^{2}}\right)\left(\frac{d H_{c 2}}{d T}\right)^{2},
$$

where $\kappa \simeq 64$ is the Ginzburg-Landau parameter at low temperatures. At temperatures near $\mathrm{T}_{c}$, the magnitude of the total specific heat is in the range 
of 15 to $20 \mathrm{~J} /$ mole $\mathrm{K}$, and the expected discontinuity is approximately 0.1 $\mathrm{J} /$ mole K. Previous measurements of the specific heat performed at LSU (chun Xu and Goodrich, 1989) using a sensitive ac technique (chun Xu, Watson, and Goodrich, 1990) did not detect a discontinuity in $C_{p}$ at the transition temperature and we believe the measurements were of sufficient accuracy to detect a $\Delta C_{p}$ of the order of magnitude predicted by Kwok et al. (1989). Recently, more high sensitivity measurements have been performed by Kim and Stewart 2 Again no discontinuity in $C_{p}$ is observed.

If one observes a second order phase transition as the normal-superconducting $(\mathrm{N}-\mathrm{S})$ phase boundary is crossed, a signature of the transition can be obtained from the temperature dependence of the thermodynamic critical field. In a second order phase transition, the free energy $F=H_{0}^{2} / 8 \pi \propto\left(1-T / T_{c}\right)^{2}$. Thus, $H_{0}(T)$ is expected to be linear in $\left(1-T / T_{c}\right)$. In Figure 6 , the measured temperature dependence of the thermodynamic critical field $\mathrm{H}_{0}$ is given with the fit to $\left(1-T / T_{c}\right)^{2}$ shown in the inset. This result clearly shows that the usual second order phase theory does not fit the data.

The experimental results for critical fields $\mathrm{H}_{c 1}, \mathrm{H}_{r}$, and $\mathrm{H}_{c 2}$ also are different from conventional superconductors in detail. More specifically, they are anomalous in subtle but important ways.

First, the GL parameter $\kappa$ as seen from equation 2 is temperature dependent (see Figure 11). In a BCS-GL framework (Tinkham, 1980), $\kappa(=\lambda / \xi)$ is temperature independent near $\mathrm{T}_{c}$. Indeed the entire classification of superconductivity between types I and II rests on a constant $\kappa$ (whether $<1 / \sqrt{2}$ or not).

We see here that $H_{c 1} \propto\left(1-T / T_{c}\right)^{3}$ and $H_{c 2} \propto\left(1-T / T_{c}\right)^{3 / 2}$, leading to $\kappa \propto\left(1-T / T_{c}\right)^{-3 / 4}$ using equation 2. The divergence of $\kappa$ near $\mathrm{T}_{c}$ indicates a much softer Meissner effect than seen in conventional superconductors.

Next, the principal premise of BCS-GL theory is a second order phase transition. The Ehrenfest relation for this second order phase transition is given by

$$
\left(\frac{d H_{c 2}}{d T}\right)^{2}=\frac{\Delta C}{T_{c} \Delta \chi},
$$

where $\Delta C$ is the discontinuity in the specific heat and $\Delta \chi$ is the discontinuity in the magnetic susceptibility. As stated above, there is evidence that $\Delta C=$ 0 . In our measurements, we find $\Delta \chi=0$.

\footnotetext{
${ }^{2}$ J. Kim and G. Stewart, University of Florida, Private Communication.
} 
It is worth noting that $\mathrm{BKBO}$, a diamagnet, is a propitious superconductor to look for non-BCS-GL effects. In a conventional superconductor, in the mixed state, the magnetic response is diamagnetic, although the magnetic susceptibility $\left(\frac{\partial M}{\partial H}\right)$ is positive, since $\mathrm{M}$ is negative. However, in the normal state most metals are weakly paramagnetic. Thus when the transition from a superconductor to a normal metal occurs, $\Delta M \neq 0$ and the transition is weakly first order. In BKBO, there is no discontinuity in M; the magnetization smoothly connects to the diamagetism of the normal state. Since both $\Delta M$ and $\Delta \chi$ appear to be zero, the order of the phase transition could be higher than two. We point out that an indication of the transition order can be obtained from the thermodynamic critical field $\mathrm{H}_{0}$. In a phase transition of order n, $F=\frac{H_{0}^{2}}{8 \pi} \propto\left(1-T / T_{c}\right)^{n}$. Thus the fit shown in Figure 6 suggests a fourth order phase transition where $H_{0} \propto\left(1-T / T_{c}\right)^{2}$.

If the transition is indeed higher than second order in the Ehrenfest sense, then, the temperature dependence of specific heat will not show a discontinuity at $\mathrm{T}_{c}$. There should be a discontinuity in the derivatives of the specific heat; however, a measurement near $\mathrm{T}_{c}$ will show effectively no sharp change in $\mathrm{C}$.

In a previous paper (Kumar, 1997), the properties of a third order phase transtion have been developed. When these same arguments are extended to a fourth order phase transition (Kumar et al., 1999) with a free energy $\mathrm{F}_{I V}$ the temperature dependance of $\mathrm{H}_{c 1}$ and $\mathrm{H}_{c 2}$ can be calculated from the free energy $\mathrm{F}_{I V}$ and the length scale $\xi^{2} \sim c / a \sim\left(1-T / T_{c}\right)^{-1}$ yeilding $H_{c 2}=\Phi_{0} / 2 \pi \xi^{2} \propto\left(1-T / T_{c}\right)$. The exponent is obtained in a mean field analysis and is lower than the observed exponent of 1.5 (1.25 near $\mathrm{T}_{c}$, see Fig. 9 caption). However, as pointed out by Gantmakher et al. (1996) the curvature in $\mathrm{H}_{c 2}$ can be enhanced by sample disorder. Next, the temperature dependence of $H_{c 1} \sim \phi_{0} / \lambda^{2} \propto\left(1-T / T_{c}\right)^{3}$. This is in agreement with the experiment; as is the corresponding $\kappa=\lambda / \xi$ in its temperature dependence.

Our conclusions about the possible order of the SC transition are published in Kumar et al. (1999); however much work still needs to be done to properly understand the nature of inhomogeneities and the effects they have on the critical magnetic fields. In addition, further analysis of fluctuations needs to be done. Thus, whether macroscopic fluctuations can lead to a different temperature dependance of the irreversibility field $\mathrm{H}_{r}$ which can influence the calculated values of $\mathrm{H}_{0}$ also remains to be analyzed. 


\section{Conclusions}

Our magnetization measurements have been carried out as field dependence at constant temperature and temperature dependence at constant field to determine the critical fields for BKBO. In all cases the agreement between the results of these two independent measurements indicate that we are measuring equilibrium properties.

From high temperature $(31$ - $350 \mathrm{~K})$ susceptibility measurements we determine the conduction electron Pauli susceptibility to be $1.8 \times 10^{-5}$ $\mathrm{emu} / \mathrm{mol}$ for two samples. The total susceptibility also contains an ostensible ferromagnetic term with a Curie-Weiss temperature near $2 \mathrm{~K}$.

We report results of the first force magnetometer measurements of BKBO to fields up to $27 \mathrm{~T}$, and show that the $\mathrm{H}_{c 2}$ and $\mathrm{H}_{r}$ critical field curves deviate from WHH theory (Werthamer et al., 1966), displaying a positive curvature at low temperatures. Our overall results including both force and SQUID measurements for these two critical fields are in general agreement with previous measurements, but show higher values of $\mathrm{H}_{c 2}$ and $\mathrm{H}_{r}$ at low temperatures than previously have been reported. We find that while fluctuation scaling theory fits the data from the SQUID measurements near $H_{c 2}$, no distinction can be made between two or three dimensional theory. This result indicates that fluctuations may not be the cause of the broadness of the transition.

Finally, we note that BKBO may exhibit a phase transition that is not of thermodynamic order two. The conclusion that the order is higher than two is based on previous measurements of the specific heat in the normal and superconducting state across the transition (no observed discontinuity), on measurements of the susceptibility across the transition (no $\Delta \chi)$, and on the fact that $\mathrm{BKBO}$ is a normal state diamagnet. The temperature dependence of the thermodynamic critical field (near $\mathrm{T}_{c}$ ), has led us to suggest that the transition is of order four.(Kumar et al., 1999) Furthermore, this is consistent with the experimental temperature dependence of $\mathrm{H}_{c 1}$ and is close to the temperature dependence of $\mathrm{H}_{c 2}$. We note that near $\mathrm{T}_{c}$, the Ginzburg-Landau parameter, $\kappa=\frac{\lambda}{\xi}$, is temperature dependent, $\kappa \sim\left(1-T / T_{c}\right)^{-3 / 4}$, a divergence which points to an unusually soft Meissner effect at $\mathrm{T}_{c}$.

Perhaps the most significant difficulty in analysis of the present data (and the data in earlier references on thermodynamic properties of BKBO) is that one's intuition is based on a tacit assumption that all superconducting transitions are second order with associated fluctuation effects. The expected properties of higher order phase transtions are different yet one tries to un- 
derstand an anomalous property in terms which implicity assume a second order phase transition. However, the evidence presented here for a higher order phase transition is by no means conclusive.

The experimental data presented here is (1) largely consistent with earlier efforts on samples of similar quality and (2) where the results are new, there may be a formalism, based on a higher order phase transition that may explain the known anomalies.

\section{Acknowledgments}

The work at LSU was supported by the NSF under grant No. DMR-9501419. A portion of this work was performed at the National High Magnetic Field Laboratory, which is supported by NSF Cooperative Agreement No. DMR9016241 and by the State of Florida. The Norton group effort was supported by the NSF under grant No. CHE-9612568.

\section{References}

M. Affronte, J. Marcus, C. Escribe-Filippini, A. Sulpice, H. Rakoto, J. M. Broto, J. C. Ousset, S. Askenazy, and A. G. M. Jansen. Upper critical field of Ba1-xKxBiO3 single crystals. Phys. Rev. B, 49(5):3502-3510, 1994.

Eduardo Alba. Automated adiabatic calorimetry, and the specific heat of Ba1-xKBi03. Master's thesis, Louisiana State University, 1992.

Aslamazov and Larkin. Phys. Lett., 26A:238, 1968.

B. Batlogg. Superconductivity in Ba(Pb, Bi)O3. Physica B, 126:275, 1984.

B. Batlogg, R. J. Cava, L. W. Rupp, A. M. Mujsce, J. J. Krajewski, J. P. Remika, W. F. Peck, A. S. a. Cooper, and G. P. Espinosa. Density of states and isotope effect in $\mathrm{BiO}$ superconductors: Evidence for nonphonon mechanism. Phys. Rev. Lett., 61:1670, 1988.

J. G. Bednorz and K. A. Müller. Possible high Tc superconductivity in the Ba-La-Cu-O system. Z. Phys. B, 64:186, 1986.

R. J. Cava, B. Batlogg, J. J. Krajewski, R. Farrow, Jr, L. W. Rupp A. E. White, K. Short, W. F. Peck, and T. Kometani. Superconductivity near 
$30 \mathrm{~K}$ without copper: the $\mathrm{Ba} 0.6 \mathrm{~K} 0.4 \mathrm{BiO} 3$ pervoskite. Nature, 332:814, 1988.

Jing chun Xu and R. G. Goodrich. 1989.

Jing chun $\mathrm{Xu}, \mathrm{C}$. H. Watson, and R. G. Goodrich. A method for measuring the specific heat of small samples. Rev. Sci. Inst., 61(2):814, 1990.

B. Dabrowski, D. G. Hinks, J. D. Jorgensen, R. K. Kalia, P. Vashishta, D. R. Richards, D. T. a. Mark, and A. W. Mitchell. Variable-range hopping conduction in Ba1-xKxBiO3-y system. Physica C, 152:24, 1988.

V. F. Gantmakher, L. A. Klinkova, N. V. Barkovskii, G. E. Tsydynzhapov, S. Wiegers, and A. K. Geim. Positive curvature in the temperature dependence of Hc2 in KxBa1-xBiO3. Phys. Rev. B, 54(9):6133-6136, Sept 1996.

Valentina I. Gatalskaya, Sergei N. Barilo, Sergei V. Shirayev, Henrick Szymezak, Rita Szymezak, and Marek Baran. Study of magnetic irreversibility line of BKBiO single crystals near Tc. Czechoslovak J. of Phys., 46:845, 1996.

G. Goll, A. G. M. Jansen, and J. Marcus. Temperature dependence of the irreversibility field of Ba1-xKxBiO3. Czechoslovak J. of Phys., 46(Suppl. S2):849, 1996.

G. S. Grader, A. F. Hebard, and L. F. Scheenmeyer. Magnetization mea-

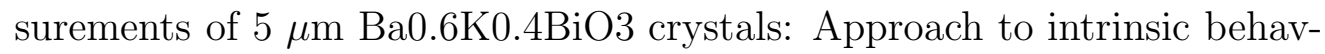
ior with decreasing size. In David Christen, Jadish Narayan, and Lynn Schneemeyer, editors, High-Temperature Superconductors: Fundamental Properties and Novel Materials Processing, volume 169 of Materials Research Society Symposium Proceedings, pages 1081 - 1084, Pittsburgh, Pennsylvania, 1990. Materials Research Society.

J. E. Graebner, L. F. Scheenmeyer, and J. K. Thomas. Heat capacity of superconducting Ba0.6K0.4BiO3 near Tc. Phys. Rev. B, 39(13):96829684, 1989.

Z. Hao, John R. Clem, M. W. McElfresh, L. Civale, A. P. Malozemoff, and F. Holtzberg. Model for the reversible magnetization of high- $\kappa$ type-II superconductors. Phys. Rev. B, 43:2844, 1991. 
D. G. Hinks, B. Dabrowski, J. D. Jorgensen, A. W. Mitchell, D. R. Richards, S. Pei, and D. Shi. Synthesis, structure and superconductivity in the Ba1xKxBiO3 system. Nature, 333:836, 1988.

Z. J. Huang, H. H. Fang, Y. Y. Xue, P. H. Hor, C. W. Chu, M. L. Norton, and H. Y. Tang. Study of the magnetic properties of single-crystal Ba0.6K0.4BiO3. Physica C, 180:331 - 336, 1991.

M. F. Hundley, J. D. Thompson, and G. H. Kwei. Specific heat of the cubic high-Tc superconductor Ba0.6K0.4BiO3. Solid State Commun., 70:1155 $-1158,1989$.

R. Itti, I. Tomeno, K. Ikeda, K. Tai, N. Koshizuka, and S. Tanaka. Photoelectron spectroscopic study of Ba1-xRbxBiO3. Phys. Rev. B, 43:435, 1991.

Pradeep Kumar. Properties of phase transitions of a higher order. J. Low Temp. Phys., 106(5/6):705-716, 1997.

Pradeep Kumar, Donavan Hall, and R. G. Goodrich. Thermodynamics of the superconducting phase transition of Ba0.6K0.4BiO3. Phys. Rev. Lett., 82(22):4532, May 1999.

W. K. Kwok, U. Welp, G. W. Crabtree, K. G. Vandervoort, R. Hulscher, Y. Zheng, B. Dabrowski, and D. G. Hinks. Magnetic and resistive measurements of the superconducting critical fields of melt-cast $\mathrm{Ba} 0.65 \mathrm{~K} 0.35 \mathrm{BiO} 3$. Phys. Rev. B, 40(13):9400-9403, 1989.

L. F. Mattheiss, E. M. Gyorgy, and D. W. Johnson Jr. Superconductivity above $20 \mathrm{~K}$ in the Ba-K-Bi-O system. Phys. Rev. B, 37(7):3745 - 3746, 1988.

L. F. Mattheiss and D. R. Hamann. Electronic structure of the high-Tc superconductor Ba1-xKxBiO3 . Phys. Rev. Lett., 60:2681, 1988.

M. E. McHenry, P. M. Maley, G. H. Kwei, and J. D. Thompson. Flux creep in a polycrystalline Ba0.6K0.4BiO3. Phys. Rev. B, 39:7339, 1989.

M. L. Norton and H-Y. Tang. Chem. Mater., 3:431-434, 1991. 
P. Samuely, P. Szabo, A. G. M. Jansen, P. Wyder, J. Marcus, L. Baril, and C. Escribe-Filippini. Upper critical magnetic field in the superconducting bismuthates studied by the point-contact spectroscopy. Czechoslovak J. of Phys., 46(Suppl. S2):847, 1996.

H. Sato, S. Tajima, H. Takagi, and S. Uchida. Optical study of the metalinsulator transition on Ba1-xKxBiO3 thin films. Nature, 338:241, 1989.

A. W. Sleight, J. L. Gilson, and P. B. Bierstedt. Solid State Commun., 17: 27, 1975.

S. E. Stupp, M. E. Reeves, D. M. Ginsberg, D. G. Hinks, B. Dabrowski, and K. G. Vandervoort. Low-temperature specific heat of polycrystalline Ba0.6K0.4BiO3. Phys. Rev. B, 40(10):10878 - 10881, 1989.

Michael Tinkham. Introduction to Superconductivity. Robert E. Krieger Publishing Company, Malabar, Florida, 2nd. edition, 1980.

Ullah and Dorsey. Phys. Rev. B, 44:262, 1991.

Hiromoto Uwe, Xiaoli Ji, and Hidetoshi Minami. Magnetic susceptibility of single crystalline Ba1-xKxBiO3. Czechoslovak J. of Phys., 46(Suppl. S5): 2691, 1996.

U. Welp, S. Fleshler, W. K. Kwok, R. A. Klemm, V. M. Vinokur, J. Downey, B. Veal, and G. W. Crabtree. High-field scaling behavior of thermodynamic and transport quantities of $\mathrm{YBa}_{2} \mathrm{Cu} 3 \mathrm{O}_{7-\delta}$ near the superconducting transition. Phys. Rev. Lett., 67(22):3180-3183, November 1991.

U. Welp, W. K. Kwok, G. W. Crabtree, H. Claus, K. G. Vandervoort, B. Dabrowski, A. W. Mitchell, D. R. Richards, D. T. Marx, and D. G. Hinks. The upper critical field of Ba1-xKxBiO3. Physica C, 156:27, 1988.

N. R. Werthamer, E. Helfand, and P. C. Hohenberg. Temperature and purity dependence of the superconducting critical field, Hc2. III Electron spin and spin-orbit effects. Phys. Rev., 147(1):295, 1966. 


\section{FIGURE CAPTIONS}

Figure 1. Complete hysteresis curve taken at 22. $5 \mathrm{~K}$ from $\mathrm{H}=0$ to 5.5 $\mathrm{T}$, back through zero to $-5.5 \mathrm{~T}$ and then to $>$ zero. An expanded view of the data showing $\mathrm{H}_{r}$ and $\mathrm{H}_{c 2}$ is given in the inset.

Figure 2. Hysteresis in the magnetization as a function of applied field of $\mathrm{Ba}_{0.6} \mathrm{~K}_{0.4} \mathrm{BiO}_{3}$ at $6.0 \mathrm{~K}$ from zero to $>+\mathrm{H}_{c 2}$ to zero. This data is the magnetization determined from the force magnetometer. $\mathrm{H}_{r}$ is indicated. The arrows show the direction of the field sweep. Note that as $\mathrm{H}$ goes to zero, the field gradient, and therefore, the force also goes to zero.

Figure 3. Example of a magnetization hysteresis taken at constant field $(\mathrm{H}=50000 \mathrm{G})$.

Figure 14. Magnetization as a function of applied field for the spherical sample of $\mathrm{Ba}_{0.6} \mathrm{~K}_{0.4} \mathrm{BiO}_{3}$ at a number of temperatures. The temperatures from from $5 \mathrm{~K}$ for the highest curve to $17.5 \mathrm{~K}$ for the lowest.

Figure 5. Values of $\mathrm{H}_{c 1}$ of $\mathrm{Ba}_{0.6} \mathrm{~K}_{0.4} \mathrm{BiO}_{3}$ as a function of temperature determined from magnetization data on the spherical sample. The inset shows that $\mathrm{H}_{c 1}$ goes as $\left(1-T / T_{c}\right)^{3}$ for temperatures higher than $12 \mathrm{~K}$.

Figure 6. Values of the thermodynamic critical field for BKBO obtained from integration of the magnetic hysteresis curves as a function of temperature. The inset shows that in the fit $\left(1-T / T_{c}\right)^{n / 2}$ that $\mathrm{n}=4$, thereby showing that the superconducting transition is of order 4 .

Figure 7. Values of $\mathrm{H}_{c 2}$ measured as a function of temperature for BKBO are shown along with the values of $\mathrm{H}_{r}$ and $\mathrm{H}_{c 2}$. $\mathrm{H}_{c 2}$ values from both constant field and constant temperature measurements are represented by a $\square$ while $\mathrm{H}_{c 2}$ values for the sample used in the force magnetometer measurements, are represented by a $\diamond$. The arrow indicates that at temperatures below $10 \mathrm{~K}$ $\mathrm{H}_{c 2}$ exceeded fields of 27 tesla, and $\mathrm{H}_{r}$ values from both constant field and constant temperature measurements are represented by a $\triangle$. The $H_{r}$ values from the force measurements are represented by a $\bowtie$. The dotted line is the phenomenological fit to other data given by Gantmakher et al. (Gantmakher et al., 1996). The inset shows the measured values of $\mathrm{H}_{c 2}(\circ), \mathrm{T}_{c 2}(\otimes), \mathrm{H}_{r}$ $(\diamond)$, and $T_{r}(\square)$ as a function of temperature.

Figure 8. The normal state susceptibility as a function of temperature for two $\mathrm{Ba}_{0.6} \mathrm{~K}_{0.4} \mathrm{BiO}_{3}$ samples. The inset shows that $\chi$ is linear in $1 / \mathrm{T}$ for 
low temperatures. The lines are a guide to the eye.

Figure 9. Temperature dependence of $\mathrm{H}_{c 2}$ and $\mathrm{H}_{r}$ from the force magnetometer and SQUID measurements combined. Note that if only the high temperature SQUID data is used, the critical exponent drops to 1.25 for $\mathrm{H}_{c 2}$.

Figure 10. $3 \mathrm{D}$ scaling of the magnetization data near $\mathrm{T}_{c} \cdot 2 \mathrm{D}$ scaling is shown in the insert.

Figure [1]. The Ginzburg-Landau parameter, $\kappa=\frac{\lambda}{\xi}$, calculated from values of $\mathrm{H}_{c 1}$ and $\mathrm{H}_{c 2}$ at selected temperatures as a function of temperature. 


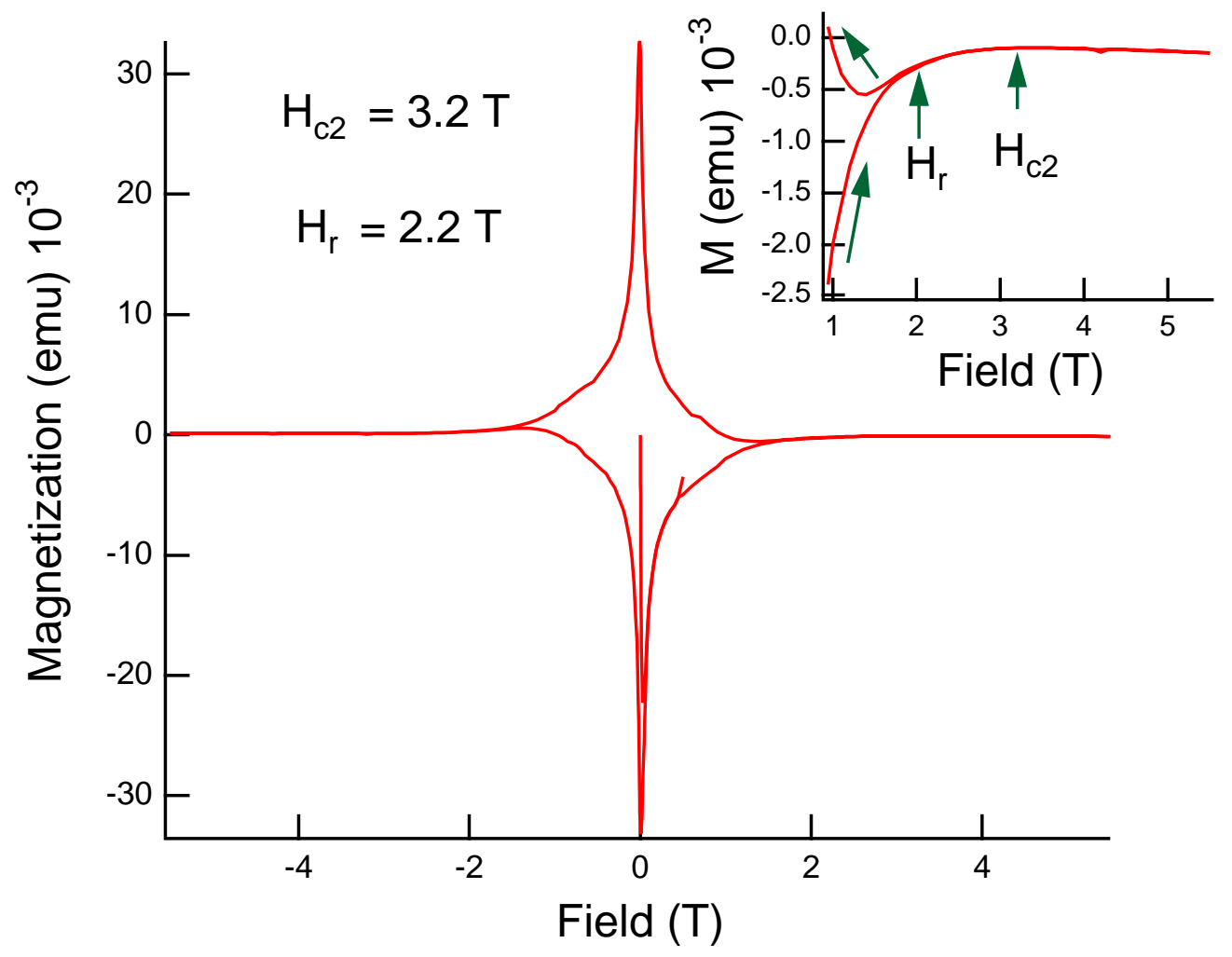

Figure 1: 


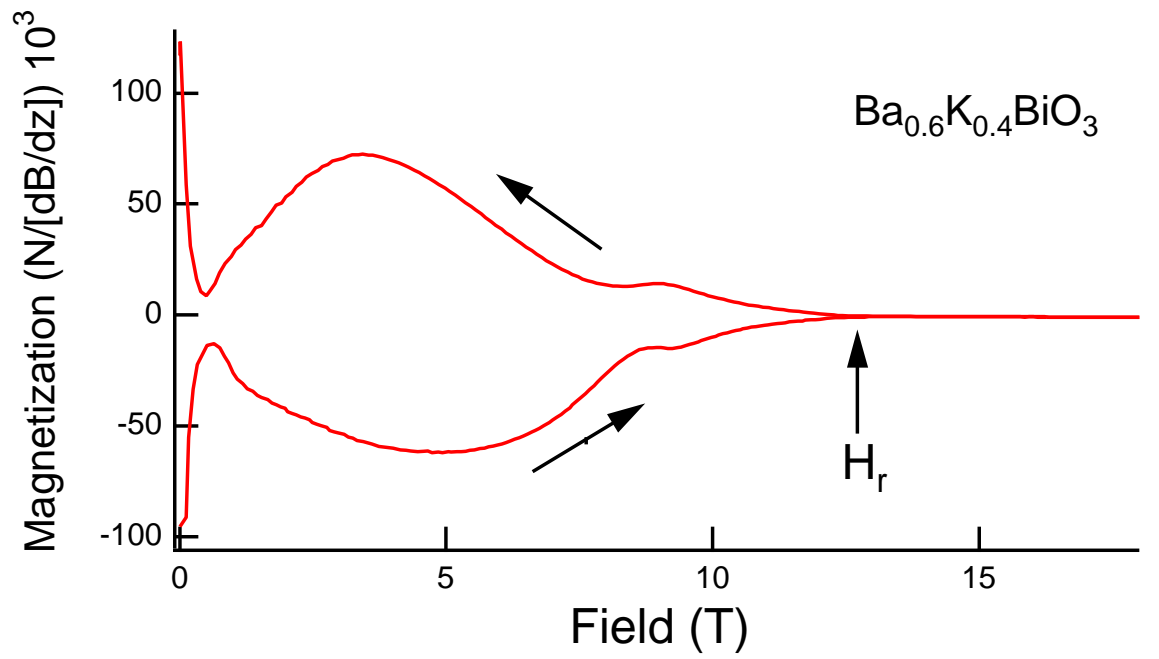

Figure 2:

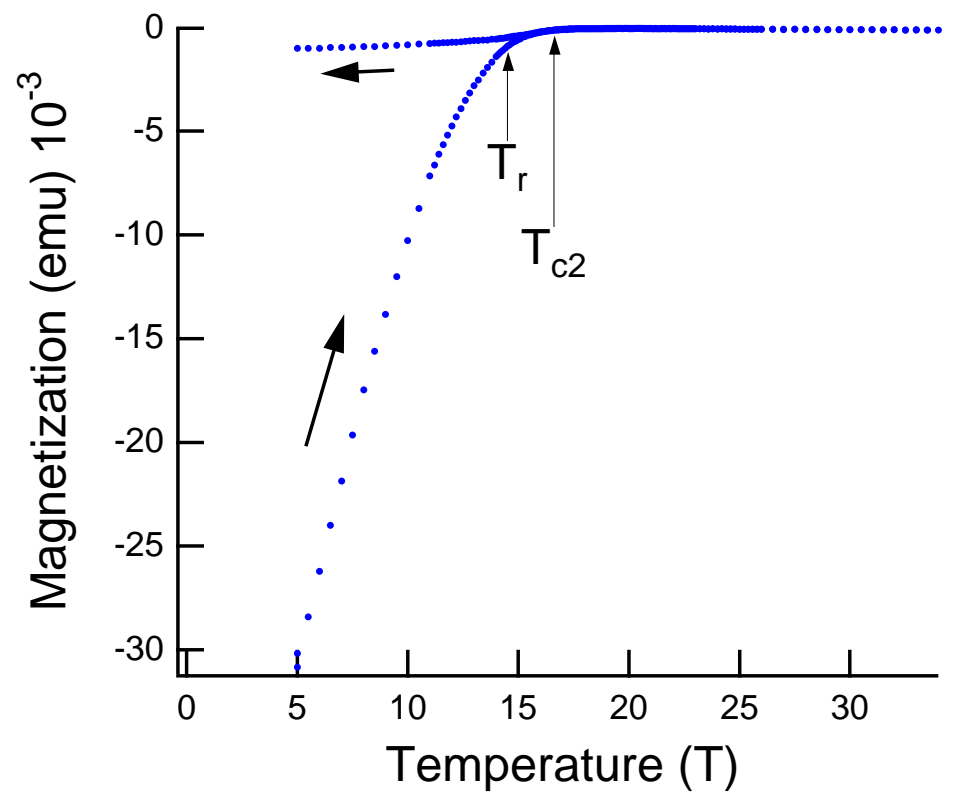

Figure 3: 


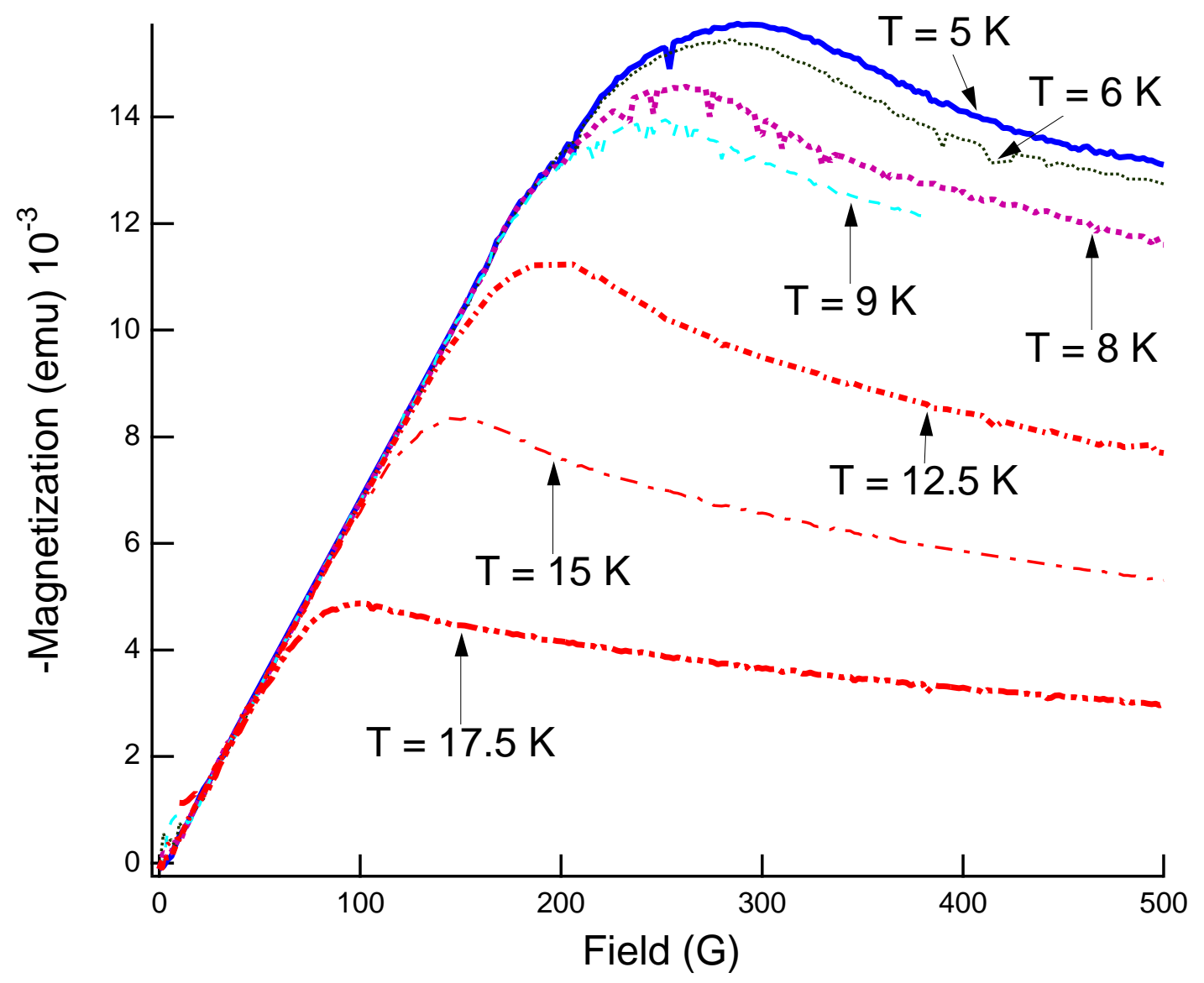

Figure 4: 


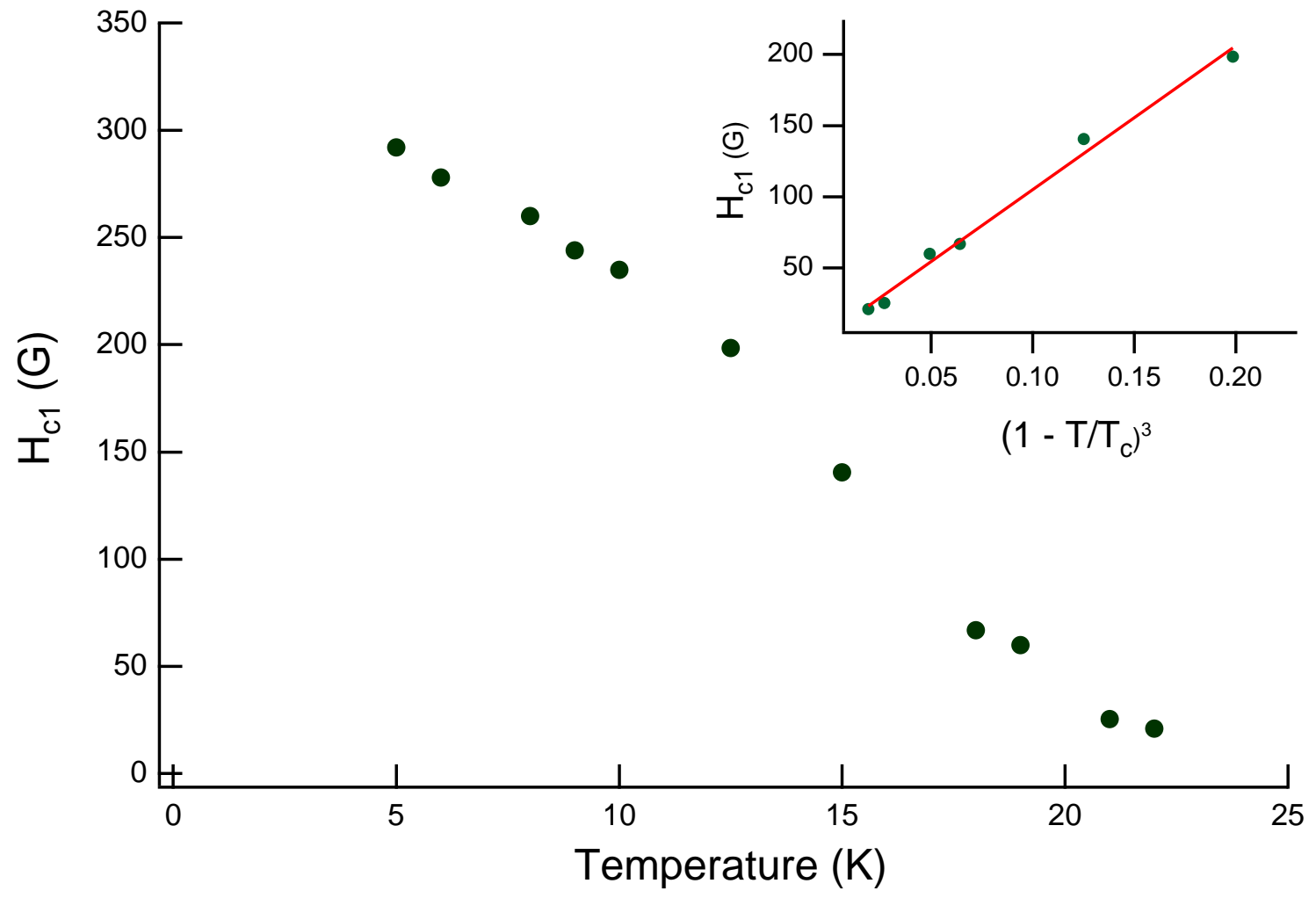

Figure 5: 


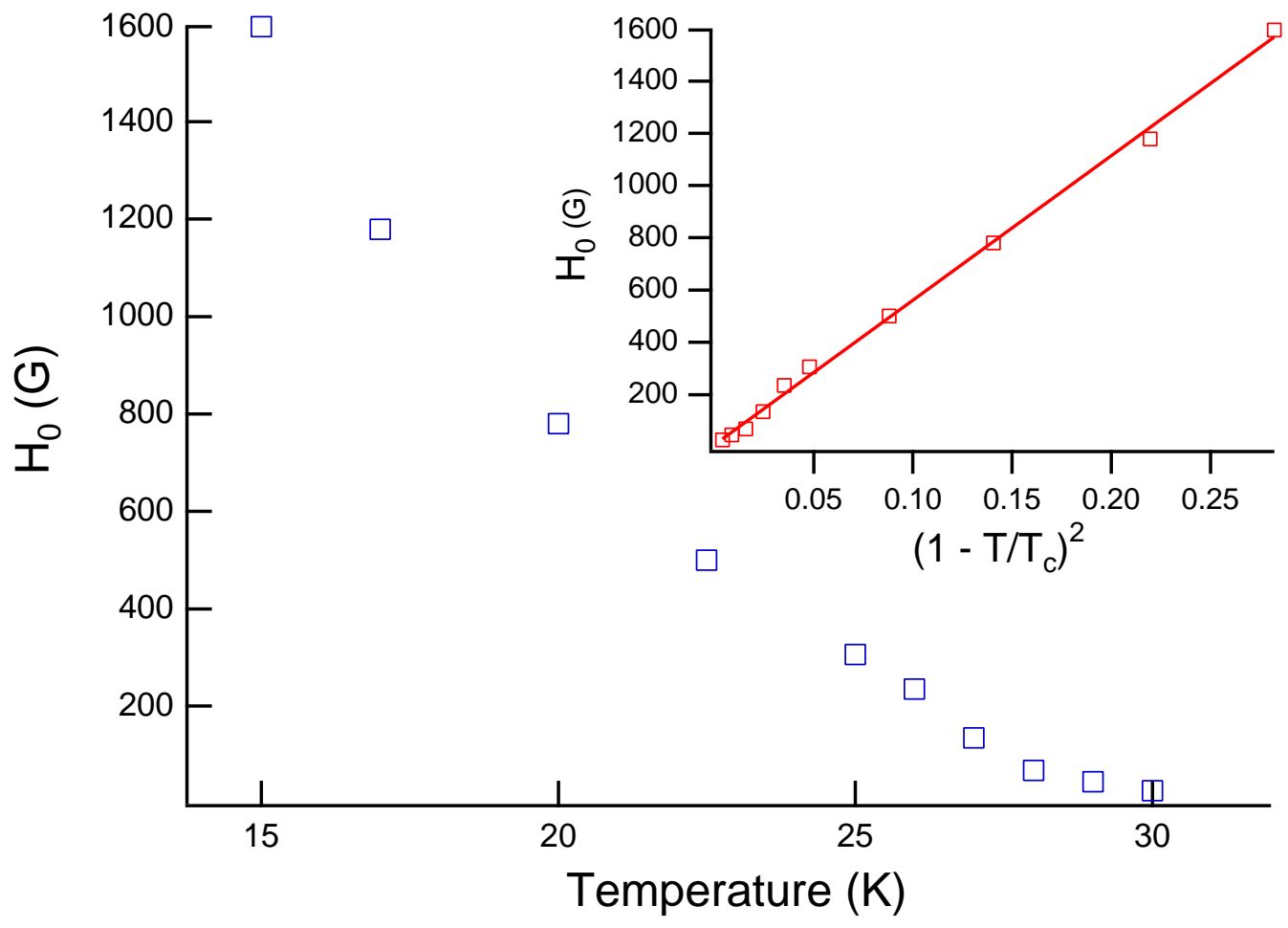

Figure 6: 


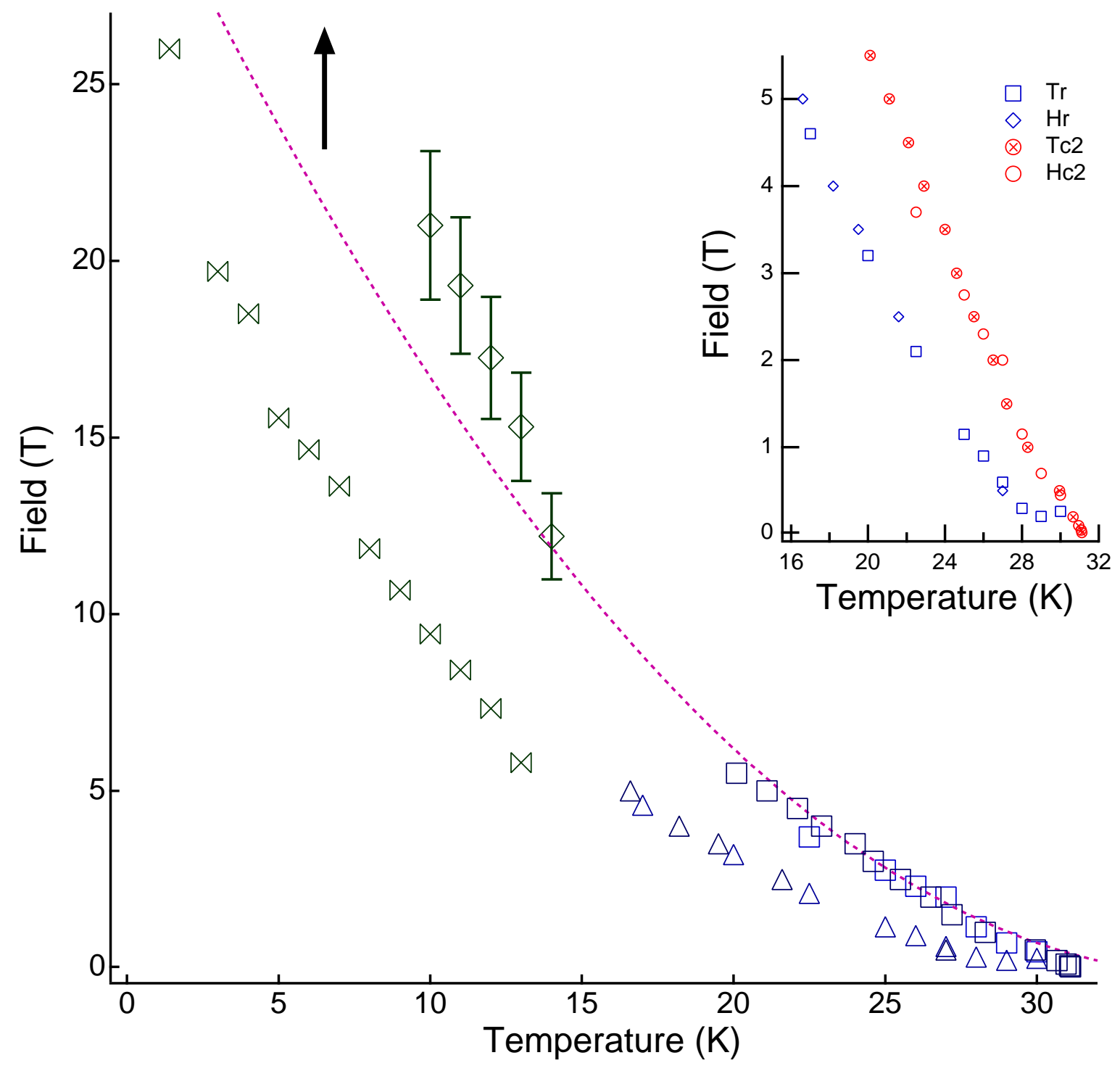

Figure 7: 


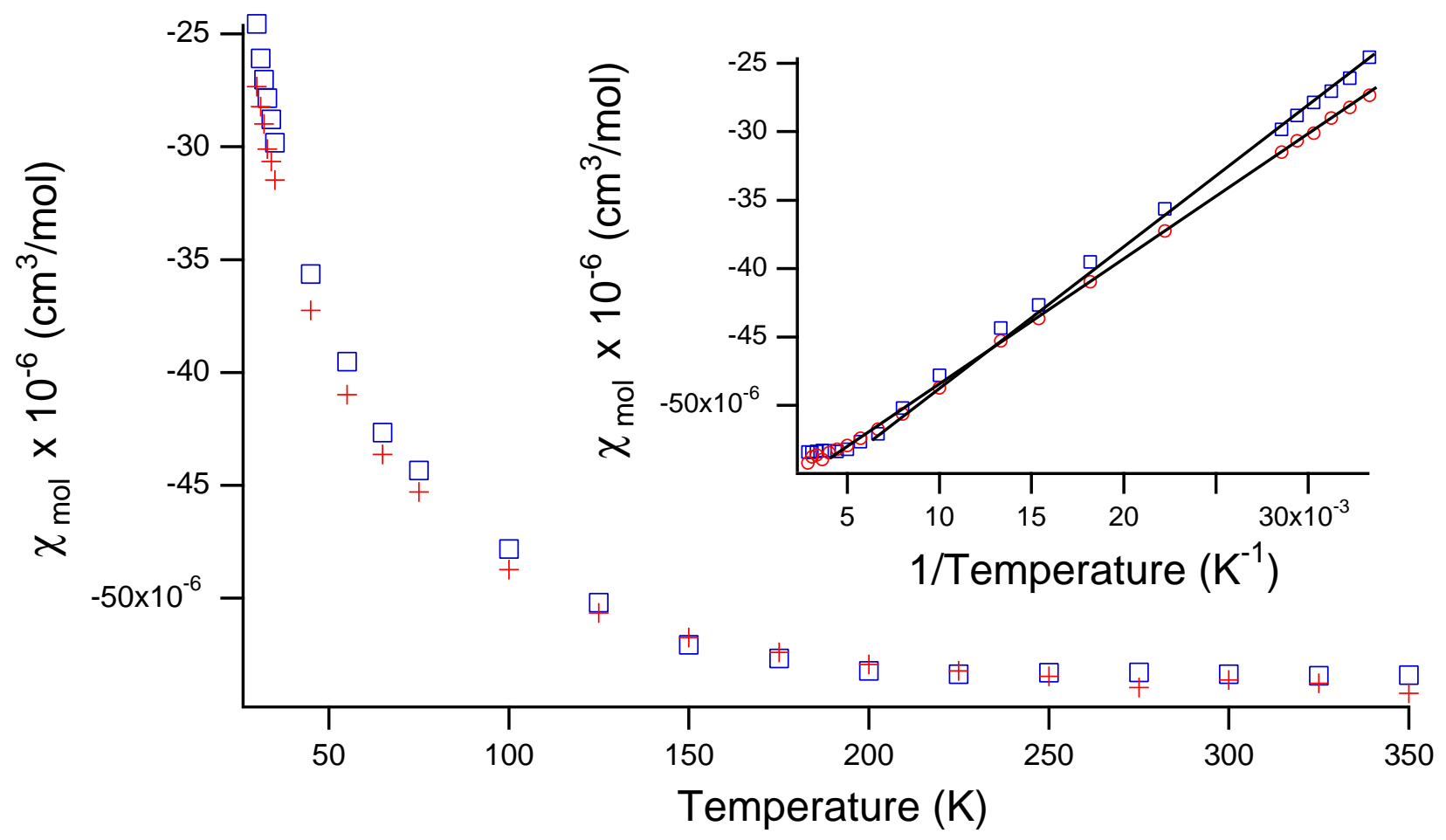

Figure 8: 


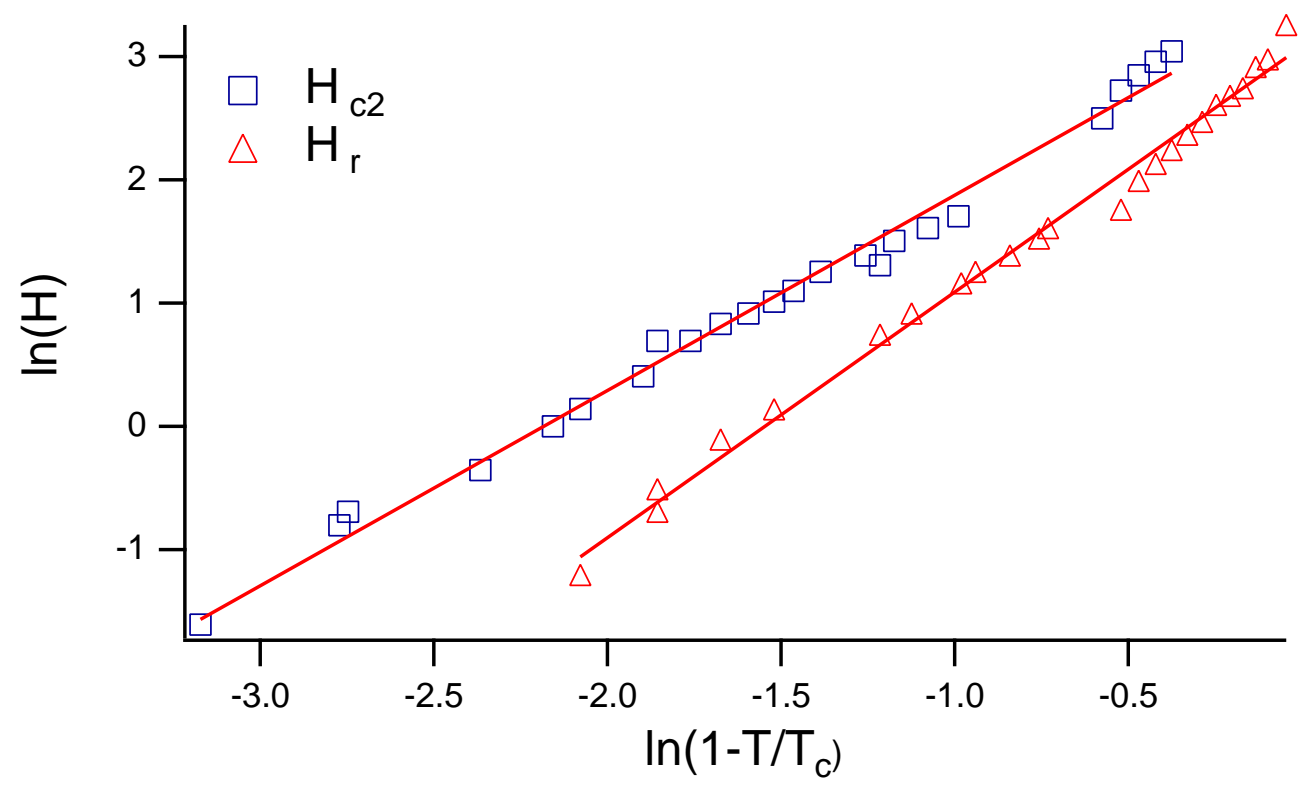

Figure 9: 


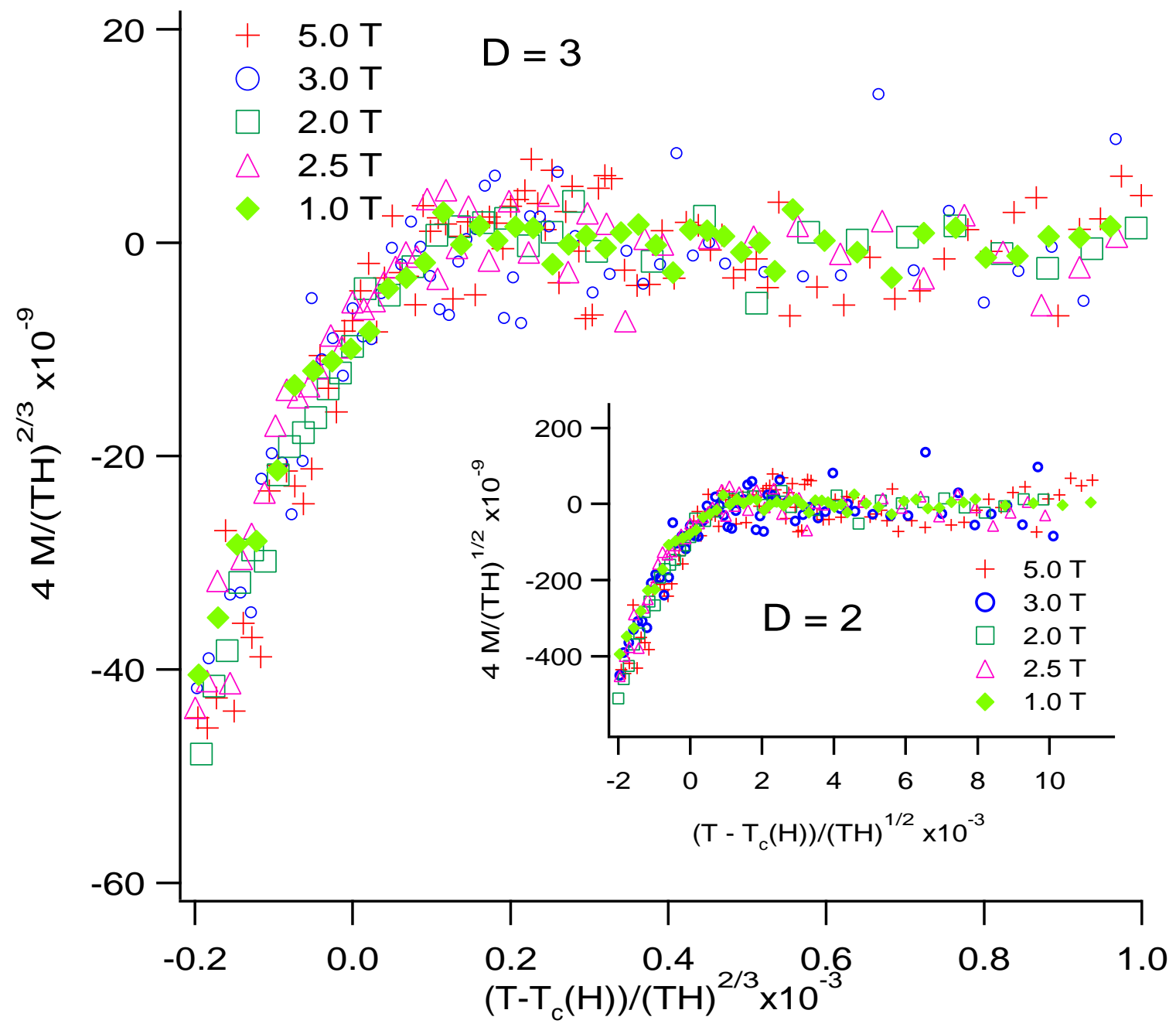

Figure 10: 


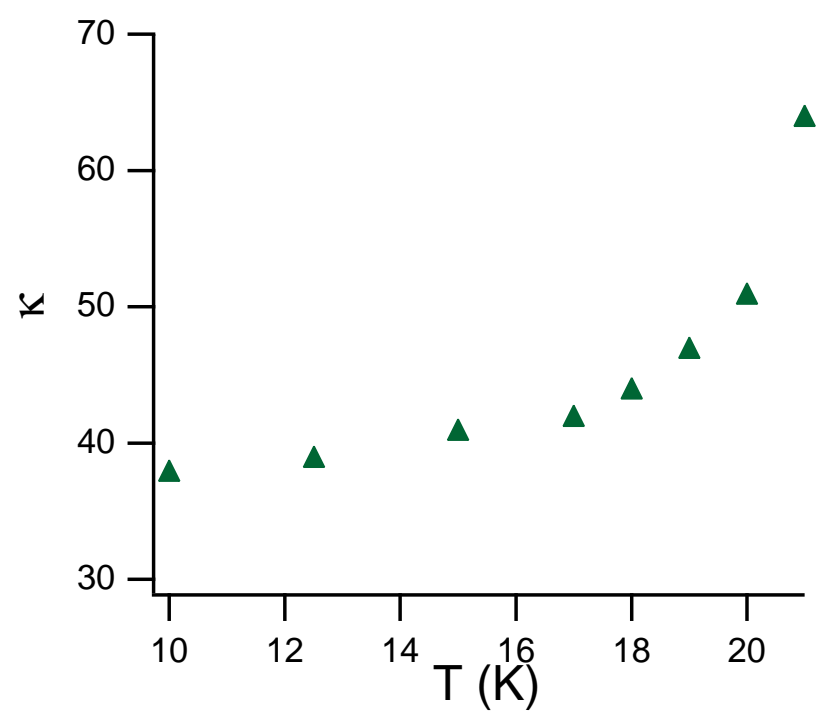

Figure 11: 